\title{
La pédagogie médicale : levier de transformation de systèmes de santé en changement
}

Nous vivons une époque historique: les systèmes de santé dans le monde sont contraints à se transformer, voire à se reconstruire, afin de donner aux différentes populations, qui sont par ailleurs de plus en plus instruites et exigeantes, des soins toujours mieux adaptés, face à des problèmes de santé dont la complexité ne cesse de grandir.

Compte tenu de l'évolution des besoins de santé et de l'évolution des sciences sous-jacentes aux pratiques des multiples professionnels qui prodiguent des soins, il est nécessaire que ces derniers s'engagent dans des activités de perfectionnement pour le maintien et le développement de leurs compétences. C'est de fait ce que nous observons, en remarquant en outre qu'un nombre grandissant de professionnels s'engagent dans des activités de perfectionnement en pédagogie. Qui l'aurait cru?

La pédagogie des formations professionnelles en santé - en indiquant par-là que la pédagogie médicale doit être comprise comme englobant la formation de l'ensemble des intervenants dans un système de santé - devient, en tant que telle, une pratique professionnelle et, par le fait même, un champ de recherche important. Il suffit de constater que la revue Pédagogie Médicale, organe officiel de la Société internationale francophone d'éducation médicale (SIFEM), et les nombreuses revues spécialisées d'expression anglaise reçoivent un nombre croissant de manuscrits de grande qualité, reflétant les multiples travaux de recherche qui se réalisent. Aucun autre domaine de pratique professionnelle ne suscite autant de publications spécialisées, mobilisant à ce point une telle communauté plurielle de chercheurs, issus à la fois de multiples disciplines et de multiples professions, engagés dans des projets de recherche éclectiques, dont les devis s'adossent à une telle variété de cadres conceptuels et méthodologiques.

Cet essor résulte d'un réel partenariat entre des chercheurs formés dans des facultés des sciences de l'éducation et des cliniciens enseignants qui se posent des questions de plus en plus pertinentes quant à leurs pratiques d'enseignement et d'évaluation. Cette synergie favorise l'obtention de subventions de recherche au service de projets innovants et aboutit à des publications académiques de grande qualité [1].

Couplé à la capacité des professionnels de la santé de s'adapter et de faire évoluer leurs pratiques de soin et d'enseignement, ce courant crée un puissant levier de transformation, qui n'a pas échappé à aucun des 300 cliniciens enseignants membres de la SIFEM, attirés dans le sillage créé par la qualité de ces travaux de recherche en pédagogie. Il est évident qu'une compréhension davantage approfondie de l'évolution de leurs professions respectives ainsi que la préparation d'une relève, apte à maintenir la qualité des services de santé prodigués aux populations, leur tiennent à cœur.

Nous constatons une remise en question des modèles classiques d'enseignement, qui se poursuit et qui s'accélère. L'innovation emblématique qu'a constituée le développement de l'«apprentissage par problèmes » par les enseignants de l'Université de McMaster ne remonte qu'aux années 1960. Elle est pourtant déjà re-questionnée en ce début de $21^{\mathrm{e}}$ siècle, notamment au regard de certaines dérives qu'elle suscite:

- transmission d'objectifs d'apprentissage de façon purement instrumentale de cohorte en cohorte, qui met à mal le principe revendiqué de co-construction des connaissances ; - variabilité de la qualité du tutorat offert qui s'avère parfois défaillant;

- maintien, malgré les intentions de départ, d'un climat de compétition qui s'accentue parmi les étudiants [2].

De tels constats, parmi de nombreux autres, motivent les enseignants à investiguer et à expérimenter d'autres perspectives et formats de dispositifs de formation, comme l'apprentissage par équipe, l'approche par compétences, la formation par concordance ou encore le mentorat avec le patient.

En effet, l'évolution des méthodes pédagogiques s'inscrit aujourd'hui dans le cadre du développement et de la mise en place d'environnements d'enseignement, d'apprentissage et d'évaluation complexes, permettant de poursuivre des objectifs élargis, qui s'efforcent désormais d'articuler les registres cognitifs, opératifs ou psycho-socio-affectifs [3]. L'essor et l'intérêt portés à la simulation participent de cet éclectisme des méthodes. Grâce à l'évolution de la technologie et aux avancées observées dans le monde de l'aéronautique - et même si l'analogie entre ce domaine et celui de l'action médicale mériterait d'être discutée de façon plus critique qu'elle ne l'est souvent-les formateurs de toutes les disciplines cliniques ont appris comment, sous certaines conditions, ils pouvaient tirer le meilleur parti de séances de simulation pour fournir des rétroactions ciblées, personnalisées et sécurisées à chacun de leurs étudiants [4].

Par ailleurs, l'évolution des dispositifs de formation interprofessionnelle vers une intégration plus grande des 
diverses ressources et expertises, témoigne de l'importance accrue, dans le contexte de réforme des systèmes de santé, de la collaboration interprofessionnelle. Il devient de moins en moins envisageable que les différents clivages séparant les différentes professions soignantes se maintiennent. Les dispositifs de formation interprofessionnelle, encore aux premiers balbutiements, sont appelés à évoluer [5].

Il va sans dire que la formation des professionnels de la santé ne peut rester en marge de l'évolution de la formation à distance et en ligne, axée sur l'apprentissage autorégulé et réflexif. De plus en plus de praticiens et chercheurs développent des dispositifs de formation utilisant des technologies qui s'efforcent d'offrir des expériences d'apprentissage plus complètes, axées sur la pratique réflexive et adaptées aux contextes particuliers des apprenants. À titre d'exemple, la formation par concordance permet aux professionnels en exercice d'accéder à un environnement d'apprentissage contextualisé et au compagnonnage cognitif sans encourir les coûts liés au déplacement. Ces innovations s'appuieront de plus en plus sur des résultats de recherche permettant d'identifier les moyens d'optimiser leur potentiel pédagogique.

En outre, ce n'est plus seulement la formation initiale qui est dans la mire. De plus en plus de travaux concernent les niveaux de la formation continue et de la formation professorale. Chacun de ces domaines deviendra un champ de pratique et de recherche en soi. Les besoins en formation continue des professionnels sont rattachés directement à l'évolution de leurs disciplines mais aussi aux besoins diversifiés de santé, ainsi qu'à l'écart grandissant entre les soins de première ligne et les soins de recours spécialisés en deuxième ou troisième ligne. La formation professorale est aussi appelée à évoluer, à ne plus se limiter à transmettre des méthodes pédagogiques, mais à inculquer le souci de développement continu des compétences d'un clinicien enseignant dans l'optique qu'il devienne un agent de changement dans son milieu de pratique et d'enseignement [6]. Ces deux champs d'activités, la formation continue et la formation professorale, sont porteurs de changements dans des milieux d'enseignement qui se transforment progressivement en de véritables communautés apprenantes.
Ainsi, à l'instar de la mutation radicale induite par le rapport Flexner, qui introduisit en Amérique du Nord les sciences biomédicales au sein de la formation des médecins, naguère artisanale, une forte remise en question des modèles classiques d'enseignement, voire des parcours de formation, est en train de se faire jour 100 ans plus tard. Notamment, nous cherchons à mettre en place des dispositifs de formation axés sur une exposition précoce et graduelle des étudiants à la pratique clinique et à toute l'incertitude qui la caractérise. Le $21^{\mathrm{e}}$ siècle est marqué d'ores et déjà par un regard critique des acquis du siècle précédent et une recherche de méthodes et dispositifs qui cherchent à promouvoir l'apprentissage autorégulé, en recourant pour cela, avec discernement, aux technologies nouvelles et à des pratiques d'évaluation des apprentissages adaptées.

Nicolas FERNANDEZ
Rédacteur en chef-adjoint

Mailto: nicolas.fernandez@umontreal.ca

\section{Références}

1. Pottier P, Buffenoir K, Castillo J-M, Etienne J, Richard I. La recherche en éducation médicale dans les facultés de médecine en France. État des lieux à partir d'une revue systématique des travaux publiés. Pédagogie Médicale 2015;16:91-103.

2. Guilbert J-J. Une vision OMS des facultés de médecine à travers le monde. Pédagogie Médicale 2004;5:167-70.

3. Vanpee D, Frenay M, Godin V, Bédard D. Ce que la perspective de l'apprentissage et de l'enseignement contextualisés authentiques peut apporter pour optimaliser la qualité pédagogique des stages d'externat. Pédagogie Médicale 2010;10:253-66.

4. Boet S, Granry J-C, Savoldelli G. La simulation en santé: De la théorie à la pratique. Paris : Springer-Verlag, 2013.

5. Fiquet L, Huge S, Annezo F, Chapron A, Allory E, Renaut P. Une formation inter professionnelle pour apprendre à travailler ensemble. La perception des étudiants en santé. Pédagogie Médicale 2015;16:105-17.

6. Mclean M, Cilliers F, Van Wyk J-M. Développement professoral: hier, aujourd'hui et demain. Guide AMEE n³6. Pédagogie Médicale 2014;15:183-237. 